\title{
A new class of general nonlinear random set- valued variational inclusion problems involving A-maximal $m$-relaxed $\eta$-accretive mappings and random fuzzy mappings in Banach spaces
}

Narin Petrot ${ }^{1}$ and Javad Balooee $2^{2^{*}}$

* Correspondence: javad. balooee@gmail.com

2Department of Mathematics, Sari Branch, Islamic Azad University,

Sari, Iran

Full list of author information is available at the end of the article

\begin{abstract}
At the present article, we consider a new class of general nonlinear random Amaximal $m$-relaxed $\eta$-accretive equations with random relaxed cocoercive mappings and random fuzzy mappings in q-uniformly smooth Banach spaces. By using the resolvent mapping technique for $A$-maximal $m$-relaxed $\eta$-accretive mappings due to Lan et al. and Chang's lemma, we construct a new iterative algorithm with mixed errors for finding the approximate solutions of this class of nonlinear random equations. We also verify that the approximate solutions obtained by the our proposed algorithm converge to the exact solution of the general nonlinear random A-maximal $m$-relaxed $\eta$-accretive equations with random relaxed cocoercive mappings and random fuzzy mappings in q-uniformly smooth Banach spaces. Mathematical Subject Classification 2010: Primary, 47B80; Secondary, 47H40, $60 \mathrm{H} 25$.
\end{abstract}

Keywords: variational inclusions, $A$-maximal $m$-relaxed $\eta$-accretive mapping, random relaxed cocoercive mapping, resolvent operator technique, random iterative algorithm, random fuzzy mapping, q-uniformly smooth Banach space

\section{Introduction}

The theory of variational inequalities was extended and generalized in many different directions because of its applications in mechanics, physics, optimization, economics and engineering sciences. For the applications, physical formulation, numerical methods and other aspects of variational inequalities (see [1-63] and the references therein). Quasi-variational inequalities are generalized forms of variational inequalities in which the constraint set depend on the solution. These were introduced and studied by Bensoussan et al. [11]. In 1991, Chang and Huang [16,17] introduced and studied some new classes of complementarity problems and variational inequalities for set-valued mappings with compact values in Hilbert spaces. An useful and important generalization of the variational inequalities is called the variational inclusions, due to Hassouni and Moudafi [34], which have wide applications in the fields of optimization and control, economics and transportation equilibrium, engineering science. 
Meanwhile, it is known that accretivity of the underlying operator plays indispensable roles in the theory of variational inequality and its generalizations. In 2001, Huang and Fang [41] were the first to introduce generalized $m$-accretive mapping and gave the definition of the resolvent operator for generalized $m$-accretive mappings in Banach spaces. Subsequently, Verma [59,60] introduced and studied new notions of $A$-monotone and $(A, \eta)$-monotone operators and studied some properties of them in Hilbert spaces. In [52], Lan et al. first introduced the concept of $(A, \eta)$-accretive mappings, which generalizes the existing $\eta$-subdifferential operators, maximal $\eta$-monotone operators, $H$-monotone operators, $A$-monotone operators, $(H, \eta)$-monotone operators, $(A, \eta)$-monotone operators in Hilbert spaces, $H$-accretive mapping, generalized $m$-accretive mappings and $(H, \eta)$-accretive mappings in Banach spaces.

On the other hand, the fuzzy set theory which was introduced by Professor Lotfi Zadeh [62] at the university of California in 1965 has emerged as an interesting and fascinating branch of pure and applied sciences. The applications of the fuzzy set theory can be found in many branches of regional, physical, mathematical and engineering sciences (see, for example $[10,32,63]$ ). In 1989 , by using the concept of fuzzy set, Chang and $\mathrm{Zu}$ [20] first introduced and studied a class of variational inequalities for fuzzy mappings. Since then several classes of variational inequalities with fuzzy mappings were considered by Chang and Haung [15], Ding [30], Ding and Park [31], Haung [36], Kumam and Petrot [48], Noor [55] and Park and Jeong [56,57] in Hilbert spaces. Recently, Huang and Lan [43], considered nonlinear equations with fuzzy mapping in fuzzy normed spaces and subsequently Lan and Verma [54] considered fuzzy variational inclusion problems in Banach spaces. It is worth to mention that variational inequalities with fuzzy mapping have been useful in the study of equilibrium and optimal control problem (see, for example [14]).

The random variational inequality and random quasi-variational inequality problems, random variational inclusion problems and random quasi-complementarity problems have been introduced and studied by Chang [13], Chang and Huang [18,19], Chang and Zhu [21], Cho et al. [22], Ganguly and Wadhawa [33], Huang and Cho [40], Khan et al. [47] and Lan [51], etc. Recently, Lan et al. [53] introduced and studied a class of general nonlinear random set-valued operator equations involving generalized $m$-accretive mappings in Banach spaces. They also established the existence theorems of the solution and convergence theorems of the generalized random iterative procedures with errors for these nonlinear random set-valued operator equations in $q$-uniformly smooth Banach spaces. Cho and Lan [23] considered and studied a class of generalized nonlinear random $(A, \eta)$-accretive equations with random relaxed cocoercive mappings in Banach spaces and by introducing some random iterative algorithms, they proved the convergence of iterative sequences generated by proposed algorithms. Further, by considering the concepts of random mappings and fuzzy mappings, Haung [39] was first introduced the concept of random fuzzy mapping. Subsequently, the random variational inclusion problem for random fuzzy mappings is studied by Ahmad and Bazan [4]. Very recently, Onjai-Uea and Kumam [58] introduced and studied a class of general nonlinear random $(H, \eta)$-accretive equations with random fuzzy mappings in Banach spaces and by using the resolvent mapping technique for the $(H, \eta)$-accretive mappings proved the existence and convergence theorems of the generalized random iterative algorithms for these nonlinear random equations with random fuzzy mappings in q-uniformly smooth Banach spaces. 
At the present article, inspired and motivated by recent researches in this field, we shall introduce and study a new class of general nonlinear random $A$-maximal $m$-relaxed $\eta$-accretive (so called $(A, \eta$ )-accretive [52]) equations with random relaxed cocoercive mappings and random fuzzy mappings in Banach spaces. By using the resolvent mapping technique for $A$-maximal $m$-relaxed $\eta$-accretive mappings due to Lan et al. [52] and Chang's lemma [12], we construct a new iterative algorithm with mixed errors for finding the approximate solutions of this class of nonlinear random equations. We also prove the existence of random solutions and the convergence of random iterative sequences generated by the our proposed algorithm in $q$-uniformly smooth Banach spaces. The results presented in this article improve and extend the corresponding results of $[13,18,22-24,33,34,37-40,42,44,46,49,53,58]$ and many other recent works.

\section{Preliminaries}

Throughout this article, let $(\Omega, \mathcal{A}, \mu)$ be a complete $\sigma$-finite measure space and $X$ be a separable real Banach space endowed with dual space $X^{*}$, the norm $\|$.$\| and the dual pair$ $\left\langle\right.$., . . between $X$ and $X^{*}$. We denote by $\mathcal{B}(X), C B(X)$ and $\hat{H}($., .) the class of Borel $\sigma$-fileds in $X$, the family of all nonempty closed bounded subsets of $X$ and the Hausdorff metric

$$
\hat{H}(A, B)=\max \left\{\sup _{x \in A} \inf _{y \in B} d(x, y), \sup _{y \in B} \inf _{x \in A} d(x, y)\right\}
$$

on $C B(X)$, respectively.

The generalized duality mapping $j_{q}: X \bowtie X^{*}$ is defined by

$$
J_{q}(x)=\left\{f^{*} \in X^{*}:\left\langle x, f^{*}\right\rangle=\|x\|^{q},\left\|f^{*}\right\|=\|x\|^{q-1}\right\}, \quad \forall x \in X,
$$

where $q>1$ is a constant. In particular, $J_{2}$ is usual normalized duality mapping. It is known that, in general, $J_{q}(x)=\|x\|^{q-2} J_{2}(x)$ for all $x \neq 0$ and $J_{q}$ is single-valued if $X^{*}$ is strictly convex. In the sequel, we always assume that $X$ is a real Banach space such that $J_{q}$ is single-valued. If $X$ is a Hilbert space, then $J_{2}$ becomes the identity mapping on $X$.

The modulus of smoothness of $X$ is the function $\rho_{X}:[0, \infty) \rightarrow[0, \infty)$ defined by

$$
\rho_{X}(t)=\sup \left\{\frac{1}{2}(\|x+y\|+\|x-y\|)-1:\|x\| \leq 1,\|y\| \leq t\right\} .
$$

A Banach space $X$ is called uniformly smooth if

$$
\lim _{t \rightarrow 0} \frac{\rho_{X}(t)}{t}=0
$$

Further, a Banach space $X$ is called q-uniformly smooth if there exists a constant $c>0$ such that

$$
\rho_{X}(t) \leq c t^{q}, \quad q>1 .
$$

It is well-known that Hilbert spaces, $L_{p}\left(\right.$ or $l_{p}$ ) spaces, $1<p<\infty$, and the Sobolev spaces $W^{m, p}, 1<p<\infty$, are all $q$-uniformly smooth. 
Concerned with the characteristic inequalities in q-uniformly smooth Banach spaces, $\mathrm{Xu}$ [61] proved the following result.

Lemma 2.1. Let $X$ be a real uniformly smooth Banach space. Then $X$ is q-uniformly smooth if and only if there exists a constant $c_{q}>0$ such that for all $x, y \in X$,

$$
\|x+y\|^{q} \leq\|x\|^{q}+q\left\langle y_{1} J_{q}(x)\right\rangle+c_{q}\|y\|^{q} .
$$

Definition 2.2. A mapping $x: \Omega \rightarrow X$ is said to be measurable if, for any $B \in \mathcal{B}(X),\{t \in \Omega: x(t) \in B\} \in \mathcal{A}$.

Definition 2.3. A mapping $T: \Omega \times X \rightarrow X$ is called a random mapping if, for any $x$ $\in X, T(., x): \Omega \rightarrow X$ is measurable. A random mapping $T$ is said to be continuous if, for any $t \in \Omega$, the mapping $T(t,):. X \rightarrow X$ is continuous.

Similarly, we can define a random mapping $a: \Omega \times X \times X \rightarrow X$. We shall write $T_{t}(x)$ $=T(t, x(t))$ and $a_{t}(x, y)=a(t, x(t), y(t))$ for all $t \in \Omega$ and $x(t), y(t) \in X$.

It is well-known that a measurable mapping is necessarily a random mapping.

Definition 2.4. A set-valued mapping $V: \Omega \bigotimes X$ is said to be measurable if, for any $B \in \mathcal{B}(X), V^{-1}(B)=\{t \in \Omega: V(t) \cap B \neq \emptyset\} \in \mathcal{A}$.

Definition 2.5. A mapping $u: \Omega \rightarrow X$ is called a measurable selection of a set-valued measurable mapping $V: \Omega \bigotimes X$ if, $u$ is measurable and for any $t \in \Omega, u(t) \in V(t)$.

Definition 2.6. A set-valued mapping $V: \Omega \times X \otimes X$ is called a random set-valued mapping if, for any $x \in X, V(., x)$ is measurable. A random set-valued mapping $V: \Omega$ $\times X \rrbracket X$ is said to be $\hat{H}$-continuous if, for any $t \in \Omega, V(t,$.$) is continuous in the Haus-$ dorff metric on $C B(X)$.

Definition 2.7. Let $X$ be a $q$-uniformly smooth Banach space, $T, A: \Omega \times X \rightarrow X$ and $\eta: \Omega \times X \times X \rightarrow X$ be random single-valued mappings. Then

(a) $T$ is said to be accretive if

$$
\left\langle T_{t}(x)-T_{t}(y), J_{q}(x(t)-y(t))\right\rangle \geq 0, \quad \forall x(t), y(t) \in X, \quad t \in \Omega ;
$$

(b) $T$ is called strictly accretive if $T$ is accretive and

$$
\left\langle T_{t}(x)-T_{t}(y), J_{q}(x(t)-y(t))\right\rangle=0,
$$

if and only if $x(t)=y(t)$ for all $t \in \Omega$;

(c) $T$ is said to be $r$-strongly accretive if there exists a measurable function $r: \Omega \rightarrow$ $(0, \infty)$ such that

$$
\left\langle T_{t}(t)-T_{t}(\gamma), J_{q}(x(t)-\gamma(t))\right\rangle \geq r(t)\|x(t)-\gamma(t)\|^{q}, \quad \forall x(t), \gamma(t) \in X, \quad t \in \Omega ;
$$

(d) $T$ is said to be $(\theta, \kappa)$-relaxed cocoercive if there exist measurable functions $\theta, \kappa$ : $\Omega \rightarrow(0, \infty)$ such that

$$
\left\langle T_{t}(x)-T_{t}(y), J_{q}(x(t)-y(t))\right\rangle \geq-\theta(t)\left\|T_{t}(x)-T_{t}(y)\right\|^{q}+\kappa(t)\|x(t)-\gamma(t)\|^{q}, \quad \forall x(t), \gamma(t) \in X, \quad t \in \Omega ;
$$

(e) $T$ is called $\mathrm{Q}$-Lipschitz continuous if there exists a measurable function $\mathrm{Q}: \Omega \rightarrow$ $(0, \infty)$ such that 


$$
\left\|T_{t}(x)-T_{t}(y)\right\| \leq \varrho(t)\|x(t)-y(t)\|, \quad \forall x(t), y(t) \in X, \quad t \in \Omega
$$

(f) $\eta$ is said to be $\tau$-Lipschitz continuous if there exists a measurable function $\tau: \Omega$ $\rightarrow(0, \infty)$ such that

$$
\left\|\eta_{t}(x, y)\right\| \leq \tau(t)\|x(t)-y(t)\|, \quad \forall x(t), y(t) \in X, \quad t \in \Omega ;
$$

(g) $\eta$ is said to be $\mu$-Lipschitz continuous in the second argument if there exists a measurable function $\mu: \Omega \rightarrow(0, \infty)$ such that

$$
\left\|\eta_{t}(x, u)-\eta_{t}(\gamma, u)\right\| \leq \mu(t)\|x(t)-\gamma(t)\|, \quad \forall x(t), \gamma(t), u(t) \in X, \quad t \in \Omega
$$

In a similar way to part ( $g$ ), we can define the Lipschitz continuity of the mapping $\eta$ in the third argument.

Definition 2.8. Let $X$ be a $q$-uniformly smooth Banach space, $\eta: \Omega \times X \times X \rightarrow X$ and $H, A: \Omega \times X \rightarrow X$ be three random single-valued mappings. Then set-valued mapping $M: \Omega \times X \rrbracket X$ is said to be:

(a) accretive if

$$
\left\langle u(t)-v(t), J_{q}(x(t)-\gamma(t))\right\rangle \geq 0, \quad \forall x(t), y(t) \in X, \quad u(t) \in M_{t}(x), \quad v(t) \in M_{t}(y), \quad t \in \Omega ;
$$

(b) $\eta$-accretive if

$$
\left\langle u(t)-v(t), J_{q}\left(\eta_{t}(x, y)\right)\right\rangle \geq 0, \quad \forall x(t), y(t) \in X, \quad u(t) \in M_{t}(x), \quad v(t) \in M_{t}(y), \quad t \in \Omega ;
$$

(c) strictly $\eta$-accretive if $M$ is $\eta$-accretive and the equality holds if and only if $x(t)=$ $y(t), \forall t \in \Omega$;

(d) $r$-strongly $\eta$-accretive if there exists a measurable function $r: \Omega \rightarrow(0, \infty)$ such that

$$
\left\langle u(t)-v(t), J_{q}\left(\eta_{t}(x, y)\right)\right\rangle \geq r(t)\|x(t)-\gamma(t)\|^{q}, \quad \forall x(t), y(t) \in X, \quad u(t) \in M_{t}(x), \quad v(t) \in M_{t}(y), \quad t \in \Omega
$$

(e) $\alpha$-relaxed $\eta$-accretive if there exists a measurable function $\alpha: \Omega \rightarrow(0, \infty)$ such that

$$
\left\langle u(t)-v(t), J_{q}\left(\eta_{t}(x, y)\right)\right\rangle \geq-\alpha(t)\|x(t)-\gamma(t)\|^{q}, \quad \forall x(t), y(t) \in X, \quad u(t) \in M_{t}(x), \quad v(t) \in M_{t}(y), \quad t \in \Omega
$$

(f) $m$-accretive if $M$ is accretive and $\left(I_{t}+\rho(t) M_{t}\right)(X)=X$ for all $t \in \Omega$ and for any measurable function $\rho: \Omega \rightarrow(0, \infty)$, where $I$ denotes the identity mapping on $X, I_{t}$ $(x)=x(t)$, for all $x(t) \in X, t \in \Omega$;

(g) generalized m-accretive if $M$ is $\eta$-accretive and $\left(I_{t}+\rho(t) M_{t}\right)(X)=X$ for all $t \in \Omega$ and any measurable function $\rho: \Omega \rightarrow(0, \infty)$;

(h) H-accretive if $M$ is accretive and $\left(H_{t}+\rho(t) M_{t}\right)(X)=X$ for all $t \in \Omega$ and any measurable function $\rho: \Omega \rightarrow(0, \infty)$, where $H_{t}()=.H(t$.) for all $t \in \Omega$;

(i) $(H, \eta)$-accretive if $M$ is $\eta$-accretive and $\left(H_{t}+\rho(t) M_{t}\right)(X)=X$ for all $t \in \Omega$ and any measurable function $\rho: \Omega \rightarrow(0, \infty)$; 
(j) A-maximal m-relaxed $\eta$-accretive if $M$ is $m$-relaxed $\eta$-accretive and $\left(A_{t}+\rho(t) M_{t}\right)$

$(X)=X$ for all $t \in \Omega$ and any measurable function $\rho: \Omega \rightarrow(0, \infty)$, where $A_{t}()=$.

$(t$.) for all $t \in \Omega$;

(k) $\beta$ - $\hat{H}$-Lipschitz continuous if there exists a measurable function $\beta: \Omega \rightarrow(0,+\infty)$ such that

$$
\hat{H}\left(M_{t}(x), M_{t}(y)\right) \leq \beta(t)\|x(t)-\gamma(t)\|, \quad \forall x(t), \gamma(t) \in X, \quad t \in \Omega .
$$

Remark 2.9. (1) If $X=\mathcal{H}$ is a Hilbert space, then parts (a)-(i) of Definition 2.8 reduce to the definitions of monotone operators, $\eta$-monotone operators, strictly $\eta$ monotone operators, strongly $\eta$-monotone operators, relaxed $\eta$-monotone operators, maximal monotone operators, maximal $\eta$-monotone operators, $H$-monotone operators and $(H, \eta)$-monotone operators, respectively.

(2) For appropriate and suitable choices of $m, A, \eta$ and $X$, it is easy to see that part (j) of Definition 2.8 includes a number of definitions of monotone operators and accretive mappings (see [52]).

Proposition 2.10. [52] Let $A: \Omega \times X \rightarrow X$ be an $r$-strongly $\eta$-accretive mapping and $M: \Omega \times X \otimes X$ be an A-maximal m-relaxed $\eta$-accretive mapping. Then the operator $\left(A_{t}+\rho(t) M_{t}\right)^{-1}$ is single-valued for any measurable function $\rho: \Omega \rightarrow(0,+\infty)$ and $t \in$ $\Omega$.

Definition 2.11. Let $A: \Omega \times X \rightarrow X$ be a strictly $\eta$-accretive mapping and $M: \Omega \times$ $X \rrbracket X$ be an $A$-maximal $m$-relaxed $\eta$-accretive mapping. Then, for any measurable function $\rho: \Omega \rightarrow(0,+\infty)$, the resolvent operator $J_{\rho(t), A_{t}}^{\eta_{t}, M_{t}}: X \rightarrow X$ is defined by:

$$
J_{\rho(t), A_{t}}^{\eta_{t}, M_{t}}(u(t))=\left(A_{t}+\rho(t) M_{t}\right)^{-1}(u(t)), \quad \forall t \in \Omega, \quad u(t) \in X .
$$

Proposition 2.12. [52] Let $X$ be a q-uniformly smooth Banach space and $\eta: \Omega \times X \times$ $X \rightarrow X$ be $\tau$-Lipschitz continuous, $A: \Omega \times X \rightarrow X$ be an $r$-strongly $\eta$-accretive mapping and $M: \Omega \times X \otimes X$ be an A-maximal m-relaxed $\eta$-accretive mapping. Then the resolvent operator $J_{\rho(t), A_{t}}^{\eta_{t}, M_{t}}: X \rightarrow X$ is $\frac{\tau^{q-1}(t)}{r(t)-\rho(t) m(t)}$-Lipschitz continuous, i.e.,

$$
\left\|J_{\rho(t), A_{t}}^{\eta_{t}, M_{t}}(x(t))-J_{\rho(t), A_{t}}^{\eta_{t}, M_{t}}(y(t))\right\| \leq \frac{\tau^{q-1}(t)}{r(t)-\rho(t) m(t)}\|x(t)-\gamma(t)\|, \quad \forall x(t), y(t) \in X, \quad t \in \Omega,
$$

where $\rho(t) \in\left(0, \frac{r(t)}{m(t)}\right)$ is a real-valued random variable for all $t \in \Omega$.

\section{A new random variational inclusion problem and random iterative algorithm}

In what follows, we denote the collection of all fuzzy sets on $X$ by $\mathfrak{F}(X)=\{A \mid A: X \rightarrow[0,1]\}$. For any set $K$, a mapping $\mathcal{S}$ from $K$ into $\mathfrak{F}(X)$ is called a fuzzy mapping. If $\mathcal{S}: K \rightarrow \mathfrak{F}(X)$ is a fuzzy mapping, then $\mathcal{S}(x)$, for any $x \in K$, is a fuzzy set on $\mathfrak{F}(X)$ (in the sequel, we denote $\mathcal{S}(x)$ by $\mathcal{S}_{x}$ ) and $\mathcal{S}_{x}(y)$, for any $y \in X$, is the degree of membership of $y$ in $\mathcal{S}_{x}$. For any $A \in \mathfrak{F}(X)$ and $\alpha \in[0,1]$, the set

$$
(A)_{\alpha}=\{x \in X: A(x) \geq \alpha\}
$$

is called a $\alpha$-cut set of $A$. 
Definition 3.1. A fuzzy mapping $\mathcal{S}: \Omega \rightarrow \mathfrak{F}(X)$ is called measurable if, for any $\alpha \in$ $(0,1],(\mathcal{S}(.))_{\alpha}: \Omega \multimap X$ is a measurable set-valued mapping.

Definition 3.2. A fuzzy mapping $\mathcal{S}: \Omega \times X \rightarrow \mathfrak{F}(X)$ is called a random fuzzy mapping if, for any $x \in X, \mathcal{S}(., x): \Omega \rightarrow \mathfrak{F}(X)$ is a measurable fuzzy mapping.

Now, let us introduce our main considered problem.

Suppose that $\mathcal{S}, \mathcal{T}, \mathcal{P}, \mathcal{Q}, \mathcal{G}: \Omega \times X \rightarrow \mathfrak{F}(X)$ are random fuzzy mappings, $A, p: \Omega \times$ $X \rightarrow X$ and $\eta: \Omega \times X \times X \rightarrow X, N: \Omega \times X \times X \times X \rightarrow X$ are random single-valued mappings. Further, let $a, b, c, d, e: X \rightarrow[0,1]$ be any mappings and $M: \Omega \times X \times X \rrbracket X$ be a random set-valued mapping such that, for each fixed $t \in \Omega$ and $z(t) \in X, M(t, ., z$ $(t)): X \otimes X$ be an $A$-maximal $m$-relaxed $\eta$-accretive mapping with $\operatorname{Im}(p)$ ndom $M(t, ., z(t)) \neq$ $\varnothing$. Now, we consider the following problem:

For any element $h: \Omega \rightarrow X$ and any measurable function $\lambda: \Omega \rightarrow(0,+\infty)$, find measurable mappings $x, v, u, v, \quad \vartheta, w: \Omega \rightarrow X$ such that for each $t \in \Omega, x(t) \in X, \mathcal{S}_{t, x(t)}(v(t)) \geq a(x(t)), \mathcal{T}_{t, x(t)}(u(t)) \geq b(x(t)), \mathcal{P}_{t, x(t)}(v(t)) \geq c(x(t)), \mathcal{Q}_{t, x(t)}(\vartheta(t)) \geq d(x(t)), \mathcal{G}_{t, x(t)}(w(t)) \geq e(x(t))$ and

$$
h(t) \in N_{t}(v, u, v)+\lambda(t) M_{t}\left(p_{t}(x)-\vartheta, w\right), \quad \forall t \in \Omega .
$$

The problem (3.1) is called the general nonlinear random A-maximal m-relaxed $\eta$ accretive equation with random relaxed cocoercive mappings and random fuzzy mappings in Banach spaces.

Remark 3.3. Obviously, the random fuzzy mapping includes set-valued mapping, random set-valued mapping and fuzzy mapping as the special cases. These mean that for appropriate and suitable choices of $X, A, \eta, \lambda, p, M, N, \mathcal{S}, \mathcal{T}, \mathcal{P}, \mathcal{Q}, \mathcal{G}$ and $h$, one can obtain many known classes of random variational inequalities, random quasivariational inequalities, random complementarity and random quasi-complementarity problems as special cases of the problem (3.1), (see, for example $[1-3,22,23,34,37,45,49,50,53,58]$ and the references therein).

In the sequel, we will develop and analyze a new class of iterative methods and construct a new random iterative algorithm with mixed errors for solving the problem (3.1). For this end, we need the following lemmas.

Lemma 3.4. [12] Let $M: \Omega \times X \rightarrow C B(X)$ be a $\hat{H}$-continuous random set-valued mapping. Then, for any measurable mapping $x: \Omega \rightarrow X$, the set-valued mapping $M(., x()$.$) :$ $\Omega \rightarrow C B(X)$ is measurable.

Lemma 3.5. [12] Let $M, V: \Omega \rightarrow C B(X)$ be two measurable set-valued mappings, $\epsilon>$ 0 be a constant and $x: \Omega \rightarrow X$ be a measurable selection of $M$. Then there exists a measurable selection $y: \Omega \rightarrow X$ of $V$ such that, for any $t \in \Omega$,

$$
\|x(t)-y(t)\| \leq(1+\varepsilon) \hat{H}(M(t), V(t)) .
$$

The following lemma offers a good approach for solving the problem (3.1).

Lemma 3.6. The set of measurable mappings $x, v, u, v, \vartheta, w: \Omega \rightarrow X$ is a random solution of the problem (3.1) if and only if, for each $t \in \Omega, v(t) \in S_{t}(x), u(t) \in T_{t}(x), v$ $(t) \in P_{t}(x), \vartheta(t) \in Q_{t}(x), w(t) \in G_{t}(x)$ and

$$
p_{t}(x)=\vartheta(t)+J_{\rho(t) \lambda(t), A_{t}}^{\eta_{t}, M_{t}(,, w)}\left[A_{t}\left(p_{t}(x)-\vartheta\right)-\rho(t)\left(N_{t}(v, u, v)-h(t)\right)\right],
$$


where $J_{\rho(t) \lambda(t), A_{t}}^{\eta_{t}, M_{t}(., w)}=\left(A_{t}+\rho(t) \lambda(t) M_{t}(., w)\right)^{-1}$ and $\rho: \Omega \rightarrow(0, \infty)$ is a measurable function.

Proof. The fact follows directly from the definition of $J_{\rho(t) \lambda(t), A_{t}}^{\eta_{t}, M_{t}(., w)}$.

In order to prove our main result, the following concepts are also needed. Let $\mathcal{S}, \mathcal{T}, \mathcal{P}, \mathcal{Q}, \mathcal{G}: \Omega \times X \rightarrow \mathfrak{F}(X)$ be five random fuzzy mappings satisfying the following condition (*): There exist five mappings $a, b, c, d, e: X \rightarrow[0,1]$ such that

$$
\begin{aligned}
& \left(\mathcal{S}_{t, x(t)}\right)_{a(x(t))} \in C B(X),\left(\mathcal{T}_{t, x(t)}\right)_{b(x(t))} \in C B(X), \quad\left(\mathcal{P}_{t, x(t)}\right)_{c(x(t))} \in C B(X) \\
& \left(\mathcal{Q}_{t, x(t)}\right)_{d(x(t))} \in C B(X),\left(\mathcal{G}_{t, x(t)}\right)_{e(x(t))} \in C B(X), \quad \forall(t, x(t)) \in \Omega \times X
\end{aligned}
$$

By using the random fuzzy mapping $\mathcal{S}$ satisfying $\left(^{*}\right)$ with the corresponding function $a: X \rightarrow[0,1]$, we can define a random set-valued mapping $S$ as follows:

$$
S: \Omega \times X \rightarrow C B(X), \quad(t, x(t)) \mapsto\left(\mathcal{S}_{t, x(t)}\right)_{a(x(t))}, \quad \forall(t, x(t)) \in \Omega \times X,
$$

Where $\mathcal{S}_{t, x(t)}=\mathcal{S}(t, x(t))$. From now on, the random fuzzy mappings $\mathcal{S}, \mathcal{T}, \mathcal{P}, \mathcal{Q}$ and $\mathcal{G}$, are assumed to satisfying the condition (*) and we will let $S, T, P, Q$ and $G$ are the random set-valued mappings induced by those five random fuzzy mappings, respectively.

Now, by using Chang's lemma [12] and based on Lemma 3.6, we can construct the new following iterative algorithm for solving the problem (3.1).

Algorithm 3.7. Let $A, p, \eta, M, N, \mathcal{S}, \mathcal{T}, \mathcal{P}, \mathcal{Q}, \mathcal{G}, h, \lambda$ be the same as in the problem (3.1) and let $S, T, P, Q, G$ be $\hat{H}$-continuous random set-valued mappings induced by $\mathcal{S}, \mathcal{T}, \mathcal{P}, \mathcal{Q}$ and $\mathcal{G}$, respectively. Assume that $\alpha: \Omega \rightarrow(0,1]$ is a measurable step size function. For any measurable mapping $x_{0}: \Omega \rightarrow X$, the set-valued mappings $S\left(., x_{0}\right.$ (.)), $T\left(., x_{0}().\right), P\left(., x_{0}().\right), Q\left(., x_{0}().\right), G\left(., x_{0}().\right): \Omega \rightarrow C B(X)$ are measurable by Lemma 3.4. Hence there exist measurable selections $v_{0}: \Omega \rightarrow X$ of $S\left(., x_{0}().\right), u_{0}: \Omega \rightarrow X$ of $T$ $\left(., x_{0}().\right), v_{0}: \Omega \rightarrow X$ of $P\left(., x_{0}().\right), \vartheta_{0}: \Omega \rightarrow X$ of $Q\left(., x_{0}().\right)$ and $w_{0}: \Omega \rightarrow X$ of $G\left(., x_{0}\right.$ (.)) by Himmelberg [35]. For each $t \in \Omega$, set

$$
\begin{aligned}
x_{1}(t)= & (1-\alpha(t)) x_{0}(t)+\alpha(t)\left\{x_{0}(t)-p_{t}\left(x_{0}\right)+\vartheta_{0}(t)+J_{\rho(t) \lambda(t), A_{t}}^{\eta_{t}, M_{t}\left(., w_{0}\right)}\left[A_{t}\left(p_{t}\left(x_{0}\right)-\vartheta_{0}\right)\right.\right. \\
& \left.\left.-\rho(t)\left(N_{t}\left(v_{0}, u_{0}, v_{0}\right)-h(t)\right)\right]\right\}+\alpha(t) e_{0}(t)+r_{0}(t),
\end{aligned}
$$

where $\rho(t)$ is the same as in Lemma 3.6 and $e_{0}, r_{0}: \Omega \rightarrow X$ are measurable functions. It is easy to know that $x_{1}: \Omega \rightarrow X$ is measurable. Since $v_{0}(t) \in S_{t}\left(x_{0}\right) \in C B(X), u_{0}(t) \in$ $T_{t}\left(x_{0}\right) \in C B(X), v_{0}(t) \in P_{t}\left(x_{0}\right) \in C B(X), \vartheta_{0}(t) \in Q_{t}\left(x_{0}\right) \in C B(X)$ and $w_{0}(t) \in G_{t}\left(x_{0}\right) \in$ $C B(X)$, by Lemma 3.5 , there exist measurable selections $v_{1}, u_{1}, v_{1}, w_{1}, \vartheta_{1}: \Omega \rightarrow X$ of the set-valued measurable mappings $S\left(., x_{1}().\right), T\left(., x_{1}().\right), P\left(., x_{1}().\right), Q\left(., x_{1}().\right)$ and $G(.$, $\left.x_{1}().\right)$, respectively, such that, for all $t \in \Omega$,

$$
\begin{aligned}
&\left\|v_{0}(t)-v_{1}(t)\right\| \leq\left(1+\frac{1}{1}\right) \hat{H}\left(S_{t}\left(x_{0}\right), S_{t}\left(x_{1}\right)\right), \\
&\left\|u_{0}(t)-u_{1}(t)\right\| \leq\left(1+\frac{1}{1}\right) \hat{H}\left(T_{t}\left(x_{0}\right), T_{t}\left(x_{1}\right)\right), \\
&\left\|v_{0}(t)-v_{1}(t)\right\| \leq\left(1+\frac{1}{1}\right) \hat{H}\left(P_{t}\left(x_{0}\right), P_{t}\left(x_{1}\right)\right), \\
&\left\|\vartheta_{0}(t)-\vartheta_{1}(t)\right\| \leq\left(1+\frac{1}{1}\right) \hat{H}\left(Q_{t}\left(x_{0}\right), Q_{t}\left(x_{1}\right)\right), \\
&\left\|w_{0}(t)-w_{1}(t)\right\| \leq\left(1+\frac{1}{1}\right) \hat{H}\left(G_{t}\left(x_{0}\right), G_{t}\left(x_{1}\right)\right) .
\end{aligned}
$$


Letting

$$
\begin{aligned}
x_{2}(t)= & (1-\alpha(t)) x_{1}(t)+\alpha(t)\left\{x_{1}(t)-p_{t}\left(x_{1}\right)+\vartheta_{1}(t)+J_{\rho(t) \lambda(t), t_{t}}^{\eta_{t}, M_{t}\left(\cdot, w_{1}\right)}\left[A_{t}\left(p_{t}\left(x_{1}\right)-\vartheta_{1}\right)\right.\right. \\
& \left.\left.-\rho(t)\left(N_{t}\left(v_{1}, u_{1}, v_{1}\right)-h(t)\right)\right]\right\}+\alpha(t) e_{1}(t)+r_{1}(t), \quad \forall t \in \Omega,
\end{aligned}
$$

then $x_{2}: \Omega \rightarrow X$ is measurable. By induction, we can define the sequences $\left\{x_{n}(t)\right\},\left\{v_{n}\right.$ $(t)\},\left\{u_{n}(t)\right\},\left\{v_{n}(t)\right\},\left\{\vartheta_{n}(t)\right\}$ and $\left\{w_{n}(t)\right\}$ for solving the problem (3.1) inductively satisfying

$$
\left\{\begin{array}{c}
x_{n+1}(t)=(1-\alpha(t)) x_{n}(t)+\alpha(t)\left\{x_{n}(t)-p_{t}\left(x_{n}\right)+\vartheta_{n}(t)+J_{\rho(t) \lambda(t), A_{t}}^{n_{t}, M_{t}\left(\cdot, w_{n}\right)}\left[A_{t}\left(p_{t}\left(x_{n}\right)-\vartheta_{n}\right)\right.\right. \\
\left.\left.\quad-\rho(t)\left(N_{t}\left(v_{n}, u_{n}, v_{n}\right)-h(t)\right)\right]\right\}+\alpha(t) e_{n}(t)+r_{n}(t), \quad \forall t \in \Omega \\
v_{n}(t) \in S_{t}\left(x_{n}\right),\left\|v_{n}(t)-v_{n+1}(t)\right\| \leq\left(1+\frac{1}{1+n}\right) \hat{H}\left(S_{t}\left(x_{n}\right), S_{t}\left(x_{n+1}\right)\right) \\
u_{n}(t) \in T_{t}\left(x_{n}\right),\left\|u_{n}(t)-u_{n+1}(t)\right\| \leq\left(1+\frac{1}{1+n}\right) \hat{H}\left(T_{t}\left(x_{n}\right), T_{t}\left(x_{n+1}\right)\right) \\
v_{n}(t) \in P_{t}\left(x_{n}\right),\left\|v_{n}(t)-v_{n+1}(t)\right\| \leq\left(1+\frac{1}{1+n}\right) \hat{H}\left(P_{t}\left(x_{n}\right), P_{t}\left(x_{n+1}\right)\right) \\
\vartheta_{n}(t) \in Q_{t}\left(x_{n}\right),\left\|\vartheta_{n}(t)-\vartheta_{n+1}(t)\right\| \leq\left(1+\frac{1}{1+n}\right) \hat{H}\left(Q_{t}\left(x_{n}\right), Q_{t}\left(x_{n+1}\right)\right) \\
w_{n}(t) \in G_{t}\left(x_{n}\right),\left\|w_{n}(t)-w_{n+1}(t)\right\| \leq\left(1+\frac{1}{1+n}\right) \hat{H}\left(G_{t}\left(x_{n}\right), G_{t}\left(x_{n+1}\right)\right)
\end{array}\right.
$$

where for all $n \geq 0$ and $t \in \Omega, e_{n}(t), r_{n}(t) \in X$ are real-valued random errors to take into account a possible inexact computation of the random resolvent operator point satisfying the following conditions:

$$
\left\{\begin{array}{l}
\lim _{n \rightarrow \infty}\left\|e_{n}(t)\right\|=\lim _{n \rightarrow \infty}\left\|r_{n}(t)\right\|=0, \quad \forall t \in \Omega \\
\sum_{n=0}^{\infty}\left\|e_{n}(t)-e_{n-1}(t)\right\|<\infty, \quad \forall t \in \Omega ; \\
\sum_{n=0}^{\infty}\left\|r_{n}(t)-r_{n-1}(t)\right\|<\infty, \quad \forall t \in \Omega
\end{array}\right.
$$

Remark 3.8. For a suitable and appropriate choice of the mappings $A, p, \eta, M, N, \mathcal{S}, \mathcal{T}, \mathcal{P}, \mathcal{Q}, \mathcal{G}, S, T, P, Q, G, \alpha, h, \lambda$, the sequences $\left\{e_{n}\right\},\left\{r_{n}\right\}$ and the space $X$, Algorithm 3.7 includes many known algorithms which due to classes of variational inequalities and variational inclusions (see, for example $[13,18,22-24,33,34,37-40,42,44,46,53,58])$.

\section{Main result}

In this section, we prove the existence of solutions for the problem (3.1) and the convergence of iterative sequences generated by Algorithm 3.7 in Banach spaces.

Theorem 4.1. Let $X$ be a q-uniformly smooth Banach space, $A, p, \eta, M, N, \mathcal{S}, \mathcal{T}, \mathcal{P}, \mathcal{Q}, \mathcal{G}, h, \lambda$ be the same as in the problem (3.1) and $S, T, P$, $Q, G: \Omega \times X \rightarrow C B(X)$ be five random set-valued mappings induced by $\mathcal{S}, \mathcal{T}, \mathcal{P}, \mathcal{Q}, \mathcal{G}$ respectively. Further, suppose that

(a) $p$ is $(\gamma, \varpi)$-relaxed cocoercive and $\pi$-Lipschitz continuous;

(b) $A$ is $r$-strongly $\eta$-accretive and $\sigma$-Lipschitz continuous;

(c) $\eta$ is $\tau$-Lipschitz continuous;

(d) $S, T, P, Q$ and $G$ are $\xi$ - $\hat{H}$-Lipschitz continuous, $\zeta$-H -Lipschitz continuous, $\varsigma-\hat{H}$ Lipschitz continuous, @- $\hat{H}$-Lipschitz continuous and $\iota-\hat{H}$-Lipschitz continuous, respectively;

(e) $N$ is $\epsilon$-Lipschitz continuous in the second argument, $\delta$-Lipschitz continuous in the third argument and $\kappa$-Lipschitz continuous in the fourth argument; 
(f) There exist the measurable functions $\mu: \Omega \rightarrow(0,+\infty)$ and $\rho: \Omega \rightarrow(0,+\infty)$ with $\rho(t) \in\left(0, \frac{r(t)}{\lambda(t) m(t)}\right)$, for all $t \in \Omega$, such that

$$
\left\|J_{\rho(t) \lambda(t), A_{t}}^{\eta_{t}, M_{t}(\cdot, x)}(z(t))-J_{\rho(t) \lambda(t), A_{t}}^{\eta_{t}, M_{t}(\cdot, y)}(z(t))\right\| \leq \mu(t)\|x(t)-y(t)\|, \quad \forall t \in \Omega, x(t), y(t), z(t) \in X
$$

and

$$
\left\{\begin{array}{l}
\varphi(t)=\varrho(t)+\mu(t) \iota(t)+\sqrt[q]{1-q \varpi(t)+\left(q \gamma(t)+c_{q}\right) \pi^{q}(t)}<1, \\
\sigma(t)(\pi(t)+\varrho(t))+\rho(t)(\varepsilon(t) \xi(t)+\delta(t) \zeta(t)+\kappa(t) \varsigma(t)) \\
<\tau^{1-q}(t)(1-\varphi(t))(r(t)-\rho(t) \lambda(t) m(t)),
\end{array}\right.
$$

where $c_{q}$ is the same as in Lemma 2.1. Then there exists a set of measurable mappings $x^{*}, v^{*}, u^{*}, v^{*}, \vartheta^{*}, w^{*}: \Omega \rightarrow X$ which is a random solution of the problem (3.1) and for each $t \in \Omega, x_{n}(t) \rightarrow x^{*}(t), v_{n}(t) \rightarrow v^{*}(t), u_{n}(t) \rightarrow u^{*}(t), v_{n}(t) \rightarrow v^{*}(t), \vartheta_{n}(t) \rightarrow \vartheta^{*}(t)$, $w_{n}(t) \rightarrow w^{*}(t)$ as $n \rightarrow \infty$, where $\left\{x_{n}(t)\right\},\left\{v_{n}(t)\right\},\left\{u_{n}(t)\right\},\left\{v_{n}(t)\right\},\left\{\vartheta_{n}(t)\right\}$ and $\left\{w_{n}(t)\right\}$ are the iterative sequences generated by Algorithm 3.7.

Proof. Firstly, for each $n \geq 0$, by considering (3.2) and (4.1), in view of Proposition 2.12 , we see that

$$
\begin{aligned}
& \left\|x_{n+1}(t)-x_{n}(t)\right\| \\
& \leq \|(1-\alpha(t)) x_{n}(t)+\alpha(t)\left\{x_{n}(t)-p_{t}\left(x_{n}\right)+\vartheta_{n}(t)+J_{\rho(t) \lambda(t), A_{t}}^{\eta_{t} M_{t}\left(f, w_{n}\right)}\left[A_{t}\left(p_{t}\left(x_{n}\right)-\vartheta_{n}\right)\right.\right. \\
& \left.\left.-\rho(t)\left(N_{t}\left(v_{n}, u_{n}, v_{n}\right)-h(t)\right)\right]\right\}+\alpha(t) e_{n}(t)+r_{n}(t)-(1-\alpha(t)) x_{n-1}(t) \\
& -\alpha(t)\left\{x_{n-1}(t)-p_{t}\left(x_{n-1}\right)+\vartheta_{n-1}(t)+J_{\rho(t) \lambda(t), A_{t}}^{n_{t}, M_{t}\left(\cdot, w_{n-1}\right)}\left[A_{t}\left(p_{t}\left(x_{n-1}\right)-\vartheta_{n-1}\right)\right.\right. \\
& \left.\left.-\rho(t)\left(N_{t}\left(v_{n-1}, u_{n-1}, v_{n-1}\right)-h(t)\right)\right]\right\}-\alpha(t) e_{n-1}(t)-r_{n-1}(t) \| \\
& \leq(1-\alpha(t))\left\|x_{n}(t)-x_{n-1}(t)\right\|+\alpha(t)\left(\left\|x_{n}(t)-x_{n-1}(t)-\left(p_{t}\left(x_{n}\right)-p_{t}\left(x_{n-1}\right)\right)\right\|\right. \\
& +\left\|\vartheta_{n}(t)-\vartheta_{n-1}(t)\right\|+\| J_{\rho(t) \lambda(t), A_{t}}^{\eta_{t}, M_{t}\left(, w_{n}\right)}\left[A_{t}\left(p_{t}\left(x_{n}\right)-\vartheta_{n}\right)-\rho(t)\left(N_{t}\left(v_{n}, u_{n}, v_{n}\right)-h(t)\right)\right] \\
& \left.-J_{\rho(t) \lambda(t), A_{t}}^{\eta_{t}, M_{t}\left(,, w_{n-1}\right)}\left[A_{t}\left(p_{t}\left(x_{n-1}\right)-\vartheta_{n-1}\right)-\rho(t)\left(N_{t}\left(v_{n-1}, u_{n-1}, v_{n-1}\right)-h(t)\right)\right] \|\right) \\
& +\alpha(t)\left\|e_{n}(t)-e_{n-1}(t)\right\|+\left\|r_{n}(t)-r_{n-1}(t)\right\| \\
& \leq(1-\alpha(t))\left\|x_{n}(t)-x_{n-1}(t)\right\|+\alpha(t)\left(\left\|x_{n}(t)-x_{n-1}(t)-\left(p_{t}\left(x_{n}\right)-p_{t}\left(x_{n-1}\right)\right)\right\|\right. \\
& +\left\|\vartheta_{n}(t)-\vartheta_{n-1}(t)\right\|+\| J_{\rho(t) \lambda(t), A_{t}}^{\eta_{t}, M_{t}\left(\cdot, w_{n}\right)}\left[A_{t}\left(p_{t}\left(x_{n}\right)-\vartheta_{n}\right)-\rho(t)\left(N_{t}\left(v_{n}, u_{n}, v_{n}\right)-h(t)\right)\right] \\
& -J_{\rho(t) \lambda(t), A_{t}}^{\eta_{t}, M_{t}\left(, w_{n}\right)}\left[A_{t}\left(p_{t}\left(x_{n-1}\right)-\vartheta_{n-1}\right)-\rho(t)\left(N_{t}\left(v_{n-1}, u_{n-1}, v_{n-1}\right)-h(t)\right)\right] \| \\
& +J_{\rho(t) \lambda(t), A_{t}}^{\eta_{t}, M_{t}\left(,, w_{t}\right)}\left[A_{t}\left(p_{t}\left(x_{n-1}\right)-\vartheta_{n-1}\right)-\rho(t)\left(N_{t}\left(v_{n-1}, u_{n-1}, v_{n-1}\right)-h(t)\right)\right] \\
& \left.-J_{\rho(t) \lambda(t), A_{t}}^{\eta_{t}, M_{t}\left(, w_{n-1}\right)}\left[A_{t}\left(p_{t}\left(x_{n-1}\right)-\vartheta_{n-1}\right)-\rho(t)\left(N_{t}\left(v_{n-1}, u_{n-1}, v_{n-1}\right)-h(t)\right)\right] \|\right) \\
& +\alpha(t)\left\|e_{n}(t)-e_{n-1}(t)\right\|+\left\|r_{n}(t)-r_{n-1}(t)\right\| \\
& \leq(1-\alpha(t))\left\|x_{n}(t)-x_{n-1}(t)\right\|+\alpha(t)\left(\left\|x_{n}(t)-x_{n-1}(t)-\left(p_{t}\left(x_{n}\right)-p_{t}\left(x_{n-1}\right)\right)\right\|+\left\|\vartheta_{n}(t)-\vartheta_{n-1}(t)\right\|\right. \\
& +\mu(t)\left\|w_{n}(t)-w_{n-1}(t)\right\|+\frac{\tau^{q-1}(t)}{r(t)-\rho(t) \lambda(t) m(t)}\left(\left\|A_{t}\left(p_{t}\left(x_{n}\right)-\vartheta_{n}\right)-A_{t}\left(p_{t}\left(x_{n-1}\right)-\vartheta_{n-1}\right)\right\|\right. \\
& \left.\left.+\rho(t)\left\|N_{t}\left(v_{t}, u_{n}, v_{n}\right)-N_{t}\left(v_{n-1}, u_{n-1}, v_{n-1}\right)\right\|\right)\right)+\alpha(t)\left\|e_{n}(t)-e_{n-1}(t)\right\|+\left\|r_{n}(t)-r_{n-1}(t)\right\| .
\end{aligned}
$$

Meanwhile, by Lemma 2.1, there exists a constant $c_{q}>0$ such that

$$
\begin{aligned}
& \left\|x_{n}(t)-x_{n-1}(t)-\left(p_{t}\left(x_{n}\right)-p_{t}\left(x_{n-1}\right)\right)\right\|^{q} \\
& \leq\left\|x_{n}(t)-x_{n-1}(t)\right\|^{q}-q\left\langle p_{t}\left(x_{n}\right)-p_{t}\left(x_{n-1}\right), J_{q}\left(x_{n}(t)-x_{n-1}(t)\right)\right\rangle+c_{q}\left\|p_{t}\left(x_{n}\right)-p_{t}\left(x_{n-1}\right)\right\|^{q} .
\end{aligned}
$$

Consequently, since $p$ is $(\gamma, \varpi)$-relaxed cocoercive and $\pi$-Lipschitz continuous, we obtain

$$
\begin{aligned}
& \left\|x_{n}(t)-x_{n-1}(t)-\left(p_{t}\left(x_{n}\right)-p_{t}\left(x_{n-1}\right)\right)\right\|^{q} \\
& \leq\left\|x_{n}(t)-x_{n-1}(t)\right\|^{q}+\left(q \gamma(t)+c_{q}\right)\left\|p_{t}\left(x_{n}\right)-p_{t}\left(x_{n-1}\right)\right\|^{q}-q \varpi(t)\left\|x_{n}(t)-x_{n-1}(t)\right\|^{q} \\
& =\left(1-q \varpi(t)+\left(q \gamma(t)+c_{q}\right) \pi^{q}(t)\right)\left\|x_{n}(t)-x_{n-1}(t)\right\|^{q} .
\end{aligned}
$$


Furthermore, by $\varrho-\hat{H}$-Lipschitz continuity of $Q$ and $\iota-\hat{H}$-Lipschitz continuity of $G$, from (3.2) we deduce that

$$
\begin{aligned}
\left\|\vartheta_{n}(t)-\vartheta_{n-1}(t)\right\| & \leq\left(1+\frac{1}{n}\right) \hat{H}\left(Q_{t}\left(x_{n}\right), Q_{t}\left(x_{n-1}\right)\right) \\
& \leq \varrho(t)\left(1+\frac{1}{n}\right)\left\|x_{n}(t)-x_{n-1}(t)\right\|
\end{aligned}
$$

and

$$
\begin{aligned}
\left\|w_{n}(t)-w_{n-1}(t)\right\| & \leq\left(1+\frac{1}{n}\right) \hat{H}\left(G_{t}\left(x_{n}\right), G_{t}\left(x_{n-1}\right)\right) \\
& \leq \iota(t)\left(1+\frac{1}{n}\right)\left\|x_{n}(t)-x_{n-1}(t)\right\| .
\end{aligned}
$$

By using (4.5) together with $\sigma$-Lipschitz continuity of $A, \pi$-Lipschitz continuity of $p$, we obtain

$$
\begin{aligned}
& \left\|A_{t}\left(p_{t}\left(x_{n}\right)-\vartheta_{n}\right)-A_{t}\left(p_{t}\left(x_{n-1}\right)-\vartheta_{n-1}\right)\right\| \\
& \leq \sigma(t)\left(\left\|p_{t}\left(x_{n}\right)-p_{t}\left(x_{n-1}\right)\right\|+\left\|\vartheta_{n}(t)-\vartheta_{n-1}(t)\right\|\right) \\
& \leq \sigma(t)\left(\pi(t)+\varrho(t)\left(1+\frac{1}{n}\right)\right)\left\|x_{n}(t)-x_{n-1}(t)\right\| .
\end{aligned}
$$

Moreover, since $N$ is $\epsilon$-Lipschitz continuous in the second argument, $\delta$-Lipschitz continuous in the third argument, $\kappa$-Lipschitz continuous in the fourth argument and $S, T, P$ are $\xi$ - $\hat{H}$-Lipschitz continuous, $\zeta$-Lipschitz continuous and $\varsigma$ - $\hat{H}$-Lipschitz continuous, respectively, by (3.2), we get

$$
\begin{aligned}
& \left\|N_{t}\left(v_{n}, u_{n}, v_{n}\right)-N_{t}\left(v_{n-1}, u_{n-1}, v_{n-1}\right)\right\| \\
& \leq\left\|N_{t}\left(v_{n}, u_{n}, v_{n}\right)-N_{t}\left(v_{n-1}, u_{n}, v_{n}\right)\right\|+\left\|N_{t}\left(v_{n-1}, u_{n}, v_{n}\right)-N_{t}\left(v_{n-1}, u_{n-1}, v_{n}\right)\right\| \\
& \quad+\left\|N_{t}\left(v_{n-1}, u_{n-1}, v_{n}\right)-N_{t}\left(v_{n-1}, u_{n-1}, v_{n-1}\right)\right\| \\
& \leq \varepsilon(t)\left\|v_{n}(t)-v_{n-1}(t)\right\|+\delta(t)\left\|u_{n}(t)-u_{n-1}(t)\right\|+\kappa(t)\left\|v_{n}(t)-v_{n-1}(t)\right\| \\
& \leq(\varepsilon(t) \xi(t)+\delta(t) \zeta(t)+\kappa(t) \zeta(t))\left(1+\frac{1}{n}\right)\left\|x_{n}(t)-x_{n-1}(t)\right\| .
\end{aligned}
$$

Now, substitute (4.4)-(4.8) into (4.3), we get that

$$
\begin{aligned}
\left\|x_{n+1}(t)-x_{n}(t)\right\| \leq & (1-\alpha(t)+\alpha(t) \psi(t, n))\left\|x_{n}(t)-x_{n-1}(t)\right\| \\
& +\alpha(t)\left\|e_{n}(t)-e_{n-1}(t)\right\|+\left\|r_{n}(t)-r_{n-1}(t)\right\|,
\end{aligned}
$$

where

$$
\begin{aligned}
& \psi(t, n)=(\varrho(t)+\mu(t) \iota(t))\left(1+\frac{1}{n}\right)+\sqrt[q]{1-q \varpi(t)+\left(q \gamma(t)+c_{q}\right) \pi^{q}(t)}+\frac{\tau^{q-1}(t) \Gamma(t, n)}{r(t)-\rho(t) \lambda(t) m(t)}, \\
& \Gamma(t, n)=\sigma(t)\left(\pi(t)+\varrho(t)\left(1+\frac{1}{n}\right)\right)+\rho(t)(\varepsilon(t) \xi(t)+\delta(t) \zeta(t)+\kappa(t) \zeta(t))\left(1+\frac{1}{n}\right) .
\end{aligned}
$$

Let us put

$$
\theta(t, n)=1-\alpha(t)+\alpha(t) \psi(t, n), \quad \text { for each } n \geq 0, \quad t \in \Omega .
$$

Then, for each $t \in \Omega$, we know that

$$
\theta(t, n) \rightarrow \theta(t)=1-\alpha(t)+\alpha(t) \psi(t), \quad \text { as } n \rightarrow \infty,
$$


where

$$
\begin{aligned}
& \psi(t)=\varrho(t)+\mu(t) \iota(t)+\sqrt[q]{1-q \varpi(t)+\left(q \gamma(t)+c_{q}\right) \pi^{q}(t)}+\frac{\tau^{q-1}(t) \Gamma(t)}{r(t)-\rho(t) \lambda(t) m(t)} \\
& \Gamma(t)=\sigma(t)(\pi(t)+\varrho(t))+\rho(t)(\varepsilon(t) \xi(t)+\delta(t) \zeta(t)+\kappa(t) \zeta(t)) .
\end{aligned}
$$

It follows that, in view of the condition (4.2), we have $\psi(t) \in(0,1)$ for all $t \in \Omega$. This implies $0<\theta(t)<1$ for all $t \in \Omega$. Hence there exist $n_{0} \in \mathbb{N}$ and a measurable function $\hat{\theta}: \Omega \rightarrow(0, \infty) \quad$ (Take $\hat{\theta}(t)=\frac{\theta(t)+1}{2} \in(\theta(t), 1)$ for each $t \in \Omega$ ) such that $\theta(t, n) \leq \hat{\theta}(t)$ for all $n \geq n_{0}$ and $t \in \Omega$. Accordingly, for all $n>n_{0}$, by (4.9), deduce that, for all $t \in \Omega$,

$$
\begin{aligned}
& \left\|x_{n+1}(t)-x_{n}(t)\right\| \\
& \leq \hat{\theta}(t)\left\|x_{n}(t)-x_{n-1}(t)\right\|+\alpha(t)\left\|e_{n}(t)-e_{n-1}(t)\right\|+\left\|r_{n}(t)-r_{n-1}(t)\right\| \\
& \leq \hat{\theta}(t)\left[\hat{\theta}(t)\left\|x_{n-1}(t)-x_{n-2}(t)\right\|+\alpha(t)\left\|e_{n-1}(t)-e_{n-2}(t)\right\|+\left\|r_{n-1}(t)-r_{n-2}(t)\right\|\right] \\
& \quad+\alpha(t)\left\|e_{n}(t)-e_{n-1}(t)\right\|+\left\|r_{n}(t)-r_{n-1}(t)\right\| \\
& =\hat{\theta}^{2}(t)\left\|x_{n-1}(t)-x_{n-2}(t)\right\|+\alpha(t)\left[\hat{\theta}(t)\left\|e_{n-1}(t)-e_{n-2}(t)\right\|\right. \\
& \left.\quad+\left\|e_{n}(t)-e_{n-1}(t)\right\|\right]+\hat{\theta}(t)\left\|r_{n-1}(t)-r_{n-2}(t)\right\|+\left\|r_{n}(t)-r_{n-1}(t)\right\| \\
& \leq \\
& \vdots \\
& \leq \\
& \quad \hat{\theta}^{n-n_{0}}(t)\left\|x_{n_{0}+1}(t)-x_{n_{0}}(t)\right\|+\sum_{i=1}^{n-n_{0}} \alpha(t) \hat{\theta}^{i-1}(t)\left\|e_{n-(i-1)}(t)-e_{n-i}(t)\right\| \\
& \quad+\sum_{i=1}^{n-n_{0}} \hat{\theta}^{i-1}(t)\left\|r_{n-(i-1)}(t)-r_{n-i}(t)\right\| .
\end{aligned}
$$

By using the inequality (4.10), it follows that, for any $m \geq n>n_{0}$,

$$
\begin{aligned}
\left\|x_{m}(t)-x_{n}(t)\right\| \leq & \sum_{j=n}^{m-1}\left\|x_{j+1}(t)-x_{j}(t)\right\| \leq \sum_{j=n}^{m-1} \hat{\theta}^{j-n_{0}}(t)\left\|x_{n_{0}+1}(t)-x_{n_{0}}(t)\right\| \\
& +\sum_{j=n}^{m-1} \sum_{i=1}^{j-n_{0}} \alpha(t) \hat{\theta}^{i-1}(t)\left\|e_{n-(i-1)}(t)-e_{n-i}(t)\right\| \\
& +\sum_{j=n}^{m-1} \sum_{i=1}^{j-n_{0}} \hat{\theta}^{i-1}(t)\left\|r_{n-(i-1)}(t)-r_{n-i}(t)\right\| .
\end{aligned}
$$

Since $\hat{\theta}(t)<1$ for all $t \in \Omega$, it follows from (3.3) and (4.11) that $\left\|x_{m}(t)-x_{n}(t)\right\| \rightarrow 0$ as $n \rightarrow \infty$. This means $\left\{x_{n}(t)\right\}$ is a Cauchy sequence in $X$. In view of completeness of $X$, there exists $x^{*}(t) \in X$ such that $x_{n}(t) \rightarrow x^{*}(t)$ for all $t \in \Omega$.

Consequently, by using (3.2), $\xi$-H-Lipschitz continuity of $S, \zeta-\hat{H}$-Lipschitz continuity of $T, \varsigma-\hat{H}$-Lipschitz continuity of $P$, Q- $\hat{H}$-Lipschitz continuity of $Q$ and $\iota-\hat{H}$-Lipschitz continuity of $G$, we know that $\left\{v_{n}(t)\right\},\left\{u_{n}(t)\right\},\left\{v_{n}(t)\right\},\left\{\vartheta_{n}(t)\right\}$ and $\left\{w_{n}(t)\right\}$ are also Cauchy sequences in $X$. Thus there are $v^{*}(t), u^{*}(t), v^{*}(t), \vartheta^{*}(t), w^{*}(t)$ in $X$ such that, for all $t \in$ $\Omega, v_{n}(t) \rightarrow v^{*}(t), u_{n}(t) \rightarrow u^{*}(t), v_{n}(t) \rightarrow v^{*}(t), v_{n}(t) \rightarrow \vartheta^{*}(t)$ and $w_{n}(t) \rightarrow w^{*}(t)$ as $n \rightarrow$ $\infty$. Since $\left\{x_{n}(t)\right\},\left\{v_{n}(t)\right\},\left\{u_{n}(t)\right\},\left\{v_{n}(t)\right\},\left\{\vartheta_{n}(t)\right\}$ and $\left\{w_{n}(t)\right\}$ are sequences of measurable mappings, we know that $x, v, u, v, \vartheta, w: \Omega \rightarrow X$ are also measurable. Further, for each $t \in \Omega$, we have 


$$
\begin{aligned}
d\left(v^{*}(t), S_{t}\left(x^{*}\right)\right) & =\inf \left\{\left\|v^{*}(t)-z\right\|: z \in S_{t}\left(x^{*}\right)\right\} \\
& \leq\left\|v^{*}(t)-v_{n}(t)\right\|+d\left(v_{n}(t), S_{t}\left(x^{*}\right)\right) \\
& \leq\left\|v^{*}(t)-v_{n}(t)\right\|+\hat{H}\left(S_{t}\left(x_{n}\right), S_{t}\left(x^{*}\right)\right) \\
& \leq\left\|v^{*}(t)-v_{n}(t)\right\|+\xi(t)\left\|x_{n}(t)-x^{*}(t)\right\| .
\end{aligned}
$$

Notice that, the right side of the above inequality tends to zero as $n \rightarrow \infty$, this implies that $v^{*}(t) \in S_{t}\left(x^{*}\right)$.

Similarly, we can verify that for each $t \in \Omega, u^{*}(t) \in T_{t}\left(x^{*}\right), v^{*}(t) \in P_{t}\left(x^{*}\right), \vartheta^{*}(t) \in Q_{t}$ $\left(x^{*}\right)$ and $w^{*}(t) \in G_{t}\left(x^{*}\right)$. Moreover, the condition (4.1) and $w_{n}(t) \rightarrow w^{*}(t)$, for all $t \in \Omega$, as $n \rightarrow \infty$, imply that for each $t \in \Omega, J_{\rho(t) \lambda(t), A_{t}}^{\eta_{t}, M_{t}\left(\cdot, w_{n}\right)} \rightarrow J_{\rho(t) \lambda(t), A_{t}}^{\eta_{t}, M_{t}\left(, w^{*}\right)}$ uniformly on $X$, as $n \rightarrow \infty$.

Now, since for each $t \in \Omega$, the mappings $J_{\rho(t) \lambda(t), A_{t}, A_{t}\left(., w_{n}\right)}$ and $A_{t}$ are continuous, it follows from (3.2) and (3.3) that for each $t \in \Omega$,

$$
p_{t}\left(x^{*}\right)=\vartheta^{*}(t)+J_{\rho(t) \lambda(t), A_{t}}^{\eta_{t} M_{t}\left(, w^{*}\right)}\left[A_{t}\left(p_{t}\left(x^{*}\right)-\vartheta^{*}\right)-\rho(t)\left(N_{t}\left(v^{*}, u^{*}, v^{*}\right)-h(t)\right)\right] .
$$

Finally, Lemma 3.6 implies that measurable mappings $x^{*}, v^{*}, u^{*}, v^{*}, 9^{*}, w^{*}: \Omega \rightarrow X$ are a random solution of the problem (3.1). This completes the proof.

Remark 4.2. If $X$ is a 2-uniformly smooth Banach space and there exists a measurable function $\rho: \Omega \rightarrow(0, \infty)$ with $\rho(t) \in\left(0, \frac{r(t)}{\lambda(t) m(t)}\right)$, for all $t \in \Omega$, such that

$$
\begin{aligned}
& \varphi(t)=\varrho(t)+\mu(t) \iota(t)+\sqrt{1-2 \varpi(t)+\left(2 \gamma(t)+c_{2}\right) \pi^{2}(t)}<1, \\
& 2 \varpi(t)-\left(2 \gamma(t)+c_{2}\right) \pi^{2}(t)<1, \\
& \rho(t)<\frac{r(t)(1-\varphi(t))-r(t) \sigma(t)(\pi(t)+\varrho(t))}{\tau(t)[(\varepsilon(t) \xi(t)+\delta(t) \zeta(t)+\kappa(t) \zeta(t)]+(1-\varphi(t)) \lambda(t) m(t)},
\end{aligned}
$$

then (4.2) holds. As we know, Hilbert spaces and $L_{p}\left(\right.$ or $\left.l_{p}\right)$ spaces, $2 \leq p<\infty$, are 2uniformly smooth.

Remark 4.3. Theorem 4.1 generalizes and improves Theorems 3.1 and 3.2 in [23], Theorems 3.1, 3.3 and 3.4 in [53] and Theorems 4.1, 4.3 and 4.4 in [58]. In brief, for an appropriate choice of the mappings $A, p, \eta, M, N, \mathcal{S}, \mathcal{T}, \mathcal{P}, \mathcal{Q}, \mathcal{G}, S, T, P, Q, G, h, \lambda$, the measurable step size function $\alpha$, the sequences $\left\{e_{n}\right\},\left\{r_{n}\right\}$ and the space $X$, Theorem 4.1 includes many known results of generalized variational inclusions as special cases (see $[13,18,22-24,33,34,37-40,42,44,46,49,53,58]$ and the references therein).

\section{Acknowledgements}

The first author was supported by the Commission on Higher Education and the Thailand Research Fund (Project No. MRG5380247).

\section{Author details \\ 'Department of Mathematics, Faculty of Science, Naresuan University, Phitsanulok 65000, Thailand ${ }^{2}$ Department of Mathematics, Sari Branch, Islamic Azad University, Sari, Iran}

Authors' contributions

Both authors contributed equally in this paper. They read and approved the final manuscript.

Competing interests

The authors declare that they have no competing interests. 


\section{References}

1. Agarwal, RP, Cho, YJ, Huang, NJ: Generalized nonlinear variational inclusions involving maximal $\eta$-monotone mappings. Nonlinear Anal Appl 1,2,59-73 (2003). (to Lakshmikantham V on His 80th Birthday. Kluwer Academic Publishers, Dordrecht, The Netherlands) 3.3

2. Agarwal, RP, Khan, MF, O'Regan, D, Salahuddin, : On generalized multivalued nonlinear variational-like inclusions with fuzzy mappings. Adv Nonlinear var Inequal 8, 41-55 (2005). 3.3

3. Ahmad, R, Ansari, QH, Irfan, SS: Generalized variational inclusions and generalized resolvent equations in Banach spaces. Comput Math Appl 49, 1825-1835 (2005). 3.3. doi:10.1016/j.camwa.2004.10.044

4. Ahmad, R, Bazan, FF: An iterative algorithm for random generalized nonlinear mixed variational inclusions for random fuzzy mappings. Appl Math Comput 167, 1400-1411 (2005). 1. doi:10.1016/j.amc.2004.08.025

5. Alimohammady, M, Balooee, J, Cho, YJ, Roohi, M: A new system of nonlinear fuzzy variational inclusions involving ( $A$,

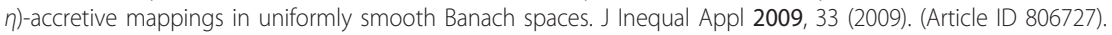
doi:10.1155/2010/806727

6. Alimohammady, M, Balooee, J, Cho, YJ, Roohi, M: Generalized nonlinear random equations with random fuzzy and relaxed cocoercive mappings in Banach spaces. Adv Nonlinear Var Inequal. 13, 37-58 (2010)

7. Alimohammady, M, Balooee, J, Cho, YJ, Roohi, M: New perturbed finite step iterative algorithms for a system of extended generalized nonlinear mixed-quasi variational inclusions. Comput Math Appl. 60, 2953-2970 (2010). doi:10.1016/j.camwa.2010.09.055

8. Balooee, J, Cho, YJ, Kang, MK: The Wiener-Hopf equation technique for solving general nonlinear regularized nonconvex variational inequalities. Fixed Point Theory Appl 2011, 86 (2011). doi:10.1186/1687-1812-2011-86. doi:10.1186/1687-1812-2011-86

9. Balooee, J, Cho, YJ, Kang, MK: Projection methods and a new system of extended general regularized nonconvex setvalued variational inequalities. J Appl Math 2012, 18 (2012). (Article ID 690648)

10. Aubin, JP: Mathematical Methods of Game and Economics Theory. North-Holland, Amsterdam (1979) 1

11. Bensoussan, A, Goursat, M, Lions, JL: Control impulsinnel et inequations quasivariationalles stationaries. C R Acad Sci 276, 1279-1248 (1973). 1

12. Chang, SS: Fixed Point Theory with Applications. Chongqing Publishing House, Chongqing (1984) 1, 3.4, 3.5, 3

13. Chang, SS: Variational Inequality and Complementarity Problem Theory with Applications. Shanghai Scientific and Tech. Literature Publishing House, Shanghai (1991). 1, 3.8, 4.3

14. Chang, MS, Chen, HY: A fuzzy user-optimal route choice problem using a link-based fuzzy variational inequality formulation. Fuzzy Sets Syst 114, 339-345 (2000). 1. doi:10.1016/S0165-0114(98)00108-0

15. Chang, SS, Huang, NJ: Generalized complementarity problems for fuzzy mappings. Fuzzy Sets Syst 55, $227-234$ (1993). 1. doi:10.1016/0165-0114(93)90135-5

16. Chang, SS, Huang, NJ: Generalized strongly nonlinear quasi-complementarity problems in Hilbert spaces. J Math Anal Appl 158, 194-202 (1991). 1. doi:10.1016/0022-247X(91)90276-6

17. Chang, SS, Huang, NJ: Generalized multivalued implicit complementarity problems in Hilbert spaces. Math Japon 36, 1093-1100 (1991). 1

18. Chang, SS, Huang, NJ: Generalized random multivalued quasi-complementarity problems. Indian J Math 35, 305-320 (1993). 1, 3.8, 4.3

19. Chang, SS, Huang, NJ: Random generalized set-valued quasi-complementarity problems. Acta Math Appl Sinica 16, 396-405 (1993). 1

20. Chang, SS, Zhu, YG: On variational inequalities for fuzzy mappings. Fuzzy Sets Syst 32, 359-367 (1989). 1. doi:10.1016/ 0165-0114(89)90268-6

21. Chang, SS, Zhu, YG: On the problems for a class of random variational inequalities and quasi-variational inequalities. J Math Res Exposition 9, 385-393 (1989). 1

22. Cho, YJ, Huang, NJ, Kang, SM: Random generalized set-valued strongly nonlinear implicit quasi-varitional inequalities. J Inequal Appl 5, 515-531 (2000). 1, 3.3, 3.8, 4.3

23. Cho, YJ, Lan, HY: Generalized nonlinear random ( $A, \eta)$-accretive equations with random relaxed cocoercive mappings in Banach spaces. Comput Math Appl 55, 2173-2182 (2008). 1, 3.3, 3.8, 4.3. doi:10.1016/j.camwa.2007.09.002

24. Cho, YJ, Petrot, N: On the system of nonlinear mixed implicit equilibrium problems in Hilbert spaces. J Inequal Appl 2010, 12 (2010). (Article ID 437976) 1, 3.8, 4.3

25. Ceng, LC, Ansari, QH, Ho, JL: Hybrid viscosity-like approximation methods for general monotone variational inequalities. Taiwanese J Math. 15, 1871-1896 (2011)

26. Ceng, LC, Ansari, QH, Yao, JC: Hybrid proximal-type and hybrid shrinking projection algorithms for equilibrium problems, maximal monotone operators, and relatively nonexpansive mappings. Numer Funct Anal Optim. 31, 763-797 (2010). doi:10.1080/01630563.2010.496697

27. Ceng, LC, Ansari, QH, Yao, JC: Hybrid pseudoviscosity approximation schemes for equilibrium problems and fixed point problems of infinitely many nonexpansive mappings. Nonlinear Anal Hybrid Syst. 4, 743-754 (2010). doi:10.1016/j. nahs.2010.05.001

28. Ceng, LC, Ansari, QH, Yao, JC: On relaxed viscosity iterative methods for variational inequalities in Banach spaces. J Comput Appl Math. 230, 813-822 (2009). doi:10.1016/j.cam.2009.01.015

29. Ceng, LC, Ansari, QH, Yao, JC: Relaxed extragradient iterative methods for variational inequalities. Appl Math Comput. 218, 1112-1123 (2011). doi:10.1016/j.amc.2011.01.061

30. Ding, XP: Algorithm of solutions for mixed implicit quasi-variational inequalities with fuzzy mappings. Comput Math Appl 38(5-6), 231-249 (1999). 1. doi:10.1016/S0898-1221(99)00229-1

31. Ding, XP, Park, JY: A new class of generalized nonlinear implicit quasivariational inclusions with fuzzy mapping. J Comput Appl Math 138, 243-257 (2002). 1. doi:10.1016/S0377-0427(01)00379-X

32. Dubois, D, Prade, H: Fuzzy Sets Systems, Theory and Applications. Academic Press, London (1980) 1

33. Ganguly, A, Wadhawa, K: On random variational inequalities. J Math Anal Appl 206, 315-321 (1997). 1, 3.8, 4.3. doi:10.1006/jmaa.1997.5194

34. Hassouni, A, Moudafi, A: A perturbed algorithm for variational inclusions. J Math Anal Appl 185, 706-712 (1994). 1, 3.3, 3.8, 4.3. doi:10.1006/jmaa.1994.1277 
35. Himmelberg, CJ: Measurable relations. Fund Math 87, 53-72 (1975). 3.7

36. Huang, NJ: A new method for a class of nonlinear variational inequalities with fuzzy mappings. Appl Math Lett 9(3), 129-133 (1997). 1

37. Huang, NJ: Generalized nonlinear variational inclusions with noncompact valued mappings. Appl Math Lett 9, 25-29 (1996). 1, 3.3, 3.8, 4.3

38. Huang, NJ: Nonlinear implicit quasi-variational inclusions involving generalized $m$-accretive mappings. Inequal Appl 2 , $413-425$ (2004). 1, 3.8, 4.3

39. Huang, NJ: Random generalized nonlinear variational inclusions for random fuzzy mappings. Fuzzy Sets Syst 105, 437-444 (1999). 1, 3.8, 4.3. doi:10.1016/50165-0114(97)00222-4

40. Huang, NJ, Cho, YJ: Random completely generalized set-valued implicit quasi-variational inequalities. Positivity 3 , 201-213 (1999). 1, 3.8, 4.3. doi:10.1023/A:1009784323320

41. Huang, NJ, Fang, YP: Generalized m-accretive mappings in Banach spaces. J Sichuan Univ 38, 591-592 (2001). 1

42. Huang, NJ, Fang, YP: A new class of general variational inclusions involving maximal $\eta$-monotone mappings. Pub Math Debrecen 62, 83-98 (2003). 1, 3.8, 4.3

43. Huang, NJ, Lan, HY: A couple of nonlinear equations with fuzzy mappings in fuzzy normed spaces. Fuzzy Sets Syst 152 209-222 (2005). 1. doi:10.1016/j.fss.2004.11.010

44. Huang, NJ, Long, X, Cho, YJ: Random completely generalized nonlinear variational inclusions with non-compact valued random mappings. Bull Korean Math Soc 34, 603-615 (1997). 1, 3.8, 4.3

45. Jeong, JU: Generalized set-valued variational inclusions and resolvent equations in Banach spaces. Comput Math Appl 47, 1241-1247 (2004). 3.3. doi:10.1016/50898-1221(04)90118-6

46. Jin, MM, Liu, QK: Nonlinear quasi-variational inclusions involving generalized $m$-accretive mappings. Non Func Anal Appl 9, 413-425 (2004). 1, 3.8, 4.3

47. Khan, MF, Salahuddin, , Verma, RU: Generalized random variational-like inequalities with randomly pseudo-monotone multivalued mappings. PanAmer Math J 16, 33-46 (2006). 1

48. Kumam, P, Petrot, N: Mixed variational-like inequality for fuzzy mappings in reflexive Banach spaces. J Inequal Appl 2009, 15 (2009). (Article ID 209485) 1

49. Lan, HY: Approximation solvability of nonlinear random $(A, \eta)$-resolvent operator equations with random relaxed cocoercive operators. Comput Math Appl 57, 624-632 (2009). 1, 3.3, 4.3. doi:10.1016/j.camwa.2008.09.036

50. Lan, HY: On multi-valued nonlinear variational inclusion problems with $(A, \eta)$-accretive mappings in Banach spaces. J Inequal Appl 2006, 12 (2006). (Article ID 59836) 3.3

51. Lan, HY: Projection iterative approximations for a new class of general random implicit quasi-variational inequalities. J Inequal Appl 2006, 17 (2006). (Article ID 81261) 1

52. Lan, HY, Cho, YJ, Verma, RU: Nonlinear relaxed cocoercive variational inclusions involving $(A, \eta)$-accretive mappings in Banach spaces. Comput Math Appl 51, 1529-1538 (2006). 1, 2.9, 2.10, 2.12. doi:10.1016/j.camwa.2005.11.036

53. Lan, HY, Cho, YJ, Xie, W: General nonlinear random equations with random multivalued operator in Banach spaces. J Inequal Appl 2009, 17 (2009). (Article ID 865093) 1, 3.3, 3.8, 4.3

54. Lan, HY, Verma, RU: Iterative algorithms for nonlinear fuzzy variational inclusion systems with (A, $\eta)$-accretive mappings in Banach spaces. Adv Nonlinear Var Inequal 11(1), 15-30 (2008). 1

55. Noor, MA: Variational inequalities with fuzzy mappings (I). Fuzzy Sets Syst 55, 309-314 (1989). 1

56. Park, JY, Jeong, JU: A perturbed algorithm of variational inclusions for fuzzy mappings. Fuzzy Sets Syst 115, 419-424 (2000). 1. doi:10.1016/50165-0114(99)00116-5

57. Park, JY, Jeong, JU: Iterative algorithm for finding approximate solutions to completely generalized strongly quasivariational inequalities for fuzzy mappings. Fuzzy Sets Syst 115, 413-418 (2000). 1. doi:10.1016/S0165-0114(98) $00339-X$

58. Onjai-Uea, N, Kumam, P: A generalized nonlinear random equations with random fuzzy mappings in uniformly smooth Banach spaces. J Inequal Appl 2010, 15 (2010). (Article ID 728452) 1, 3.3, 3.8, 4.3

59. Verma, RU: A-monotonicity and applications to nonlinear inclusion problems. J Appl Math Stoch Anal 17, 193-195 (2004). 1

60. Verma, RU: The over-relaxed A-proximal point algorithm and applications to nonlinear variational inclusions in Banach spaces. Fixed Point Theory 10, 185-195 (2009). 1

61. Xu, HK: Inequalities in Banach spaces with applications. Nonlinear Anal 16, 1127-1138 (1991). 2. doi:10.1016/0362-546X (91)90200-K

62. Zadeh, LA: Fuzzy sets. Inf Control 8, 338-358 (1965). 1. doi:10.1016/S0019-9958(65)90241-X

63. Zimmermann, HI: Fuzzy Set Theory and its Applications. Kluwer Academic Publishing Group, Boston, MA (1988) 1

doi:10.1186/1029-242X-2012-98

Cite this article as: Petrot and Balooee: A new class of general nonlinear random set-valued variational inclusion problems involving $A$-maximal $m$-relaxed $\eta$-accretive mappings and random fuzzy mappings in Banach spaces. Journal of Inequalities and Applications 2012 2012:98. 